\title{
Efficiency Comparison in Power Converters under Transient Operation Conditions: Application to Hybrid Energy Storage Systems
}

\author{
Jorge García, Cristina González-Morán, Pablo García Fernández, Pablo Arboleya \\ LEMUR Research Group \\ Dept. Electrical Engineering, Univeristy of Oviedo, \\ Gijon, Spain \\ garciajorge@uniovi.es
}

\begin{abstract}
This work proposes a methodology for selecting the optimal configuration, in terms of energy efficiency, in power conversion applications. The outcomes of the selection are both the power topology among different options, together with a set of parameters for the design variables for that topology ensuring the highest efficiency for the target operating conditions. An example of this methodology is provided for a Hybrid Energy Storage System application in a given nanogrid. Two options for the power converter topology are presented, and also each of them is assessed in two basic operation modes: islanding mode and transient support mode. The final methodology provides a tool for selecting among the optimal power topology design considering the total efficiency of the system.
\end{abstract}

Keywords-Power converters, efficiency, transient operation, hybrid energy storage.

\section{INTRODUCTION}

The analysis of the efficiency in power converters is a complex task that depends largely on the application and ratings, the operating conditions, the accuracy of the analytical and simulation models considered, etc. [1]-[4]. The basic methodology characterizes the efficiency $\left(\eta_{d}\right)$ of a any general system able to process energy, as a function of the steady-state average rated input and output power values $\left(P_{I}\right.$ and $P_{O}$, respectively), as given by:

$$
\eta_{d}=\frac{P_{O}}{P_{I}}
$$

This definition is usually given for the operation of power electronic converters, in steady state, at nominal operating values. Therefore, even though it gives a general idea of the performance of the system, this figure of merit does not take into account the evolution of the system during transient or complex operation modes, in which steady state is not clearly defined (e.g. in an energy backup system the steady state is idle, thus the output power is null most of the time).

In general terms, a power converter might operate at conditions well away from the rated settings. Examples of

This work has been partially supported by the Spanish Government, Innovation Development and Research Office (MEC), under research grant ENE2016-77919, Project "Conciliator", and by the European Union through ERFD Structural Funds (FEDER). This work has been partially supported by the government of Principality of Asturias, under IDEPA grant 2017 Thyssen SV-PA-17-RIS3-3 applications are Photovoltaic (PV) systems (with varying irradiance), powertrain converters in Electric Vehicles (EVs) (with varying load profiles), storage systems in microgrids (with random load and generation profiles), etc. [1]-[10].

In these applications, the standard definition of efficiency (steady state, nominal operation) might be not enough as to take design decisions based on the energy efficiency performance. A way to cope with this issue in PV applications, several complex efficiency definitions have been proposed, such as the European Efficiency $\left(\eta_{E}\right)$ or the California Energy Commission Efficiency $\left(\eta_{C}\right)$ [11]-[12]. These are weighted average values of the converter efficiency calculated from the efficiency values measured at different loads:

$$
\begin{gathered}
\eta_{E}=0.03 \eta_{5 \%}+0.06 \eta_{10 \%}+0.13 \eta_{20 \%}+ \\
\quad+0.1 \eta_{30 \%}+0,48 \eta_{50 \%}+0.2 \eta_{100 \%} \\
\eta_{C}=0.04 \eta_{10 \%}+0.05 \eta_{20 \%} 0.12 \eta_{30 \%}+ \\
+0.21 \eta_{50 \%}+0.53 \eta_{75 \%}+0.05 \eta_{100 \%}
\end{gathered}
$$

being $\eta_{i \%}$ the efficiency of the converter at a percentage $i \%$ of the load of the rated power value.

These weighted efficiencies account for the converter performance in a wide range of loads, and are good parameters in order to compare different inverters for a given PV installation [13]-[15]. However, in the case of applications in which the main operation is intended to be a sequence of transients rather than a steady state operation point (e.g. transportation, stochastic loads, microgrid storage systems, etc.), the definition of efficiency as a quotient between input and output steady state average power values does not provide enough information of the performance of the energy conversion.

This work proposes a methodology for selecting the optimal configuration in power conversion systems, in terms of energy efficiency. The optimal configuration is defined as a set of parameters of given design variables (such as specific power converter topology choice among different options, values of control parameters as for instance the bandwidth of the controllers, etc.) that ensure the highest efficiency in given operating conditions. These operating conditions are defined as a number of different cases of study. This work firstly defines the methodology, and later provides a case study in a particular application of a Hybrid Energy Storage system (HESS) in a nanogrid. The modes of operation of such HESS considered are islanding mode (HESS supplying power to all the loads at the nanogrid and controlling the DC bus at the nanogrid), and transient support (nanogrid connected in grid-tied mode and 
controlling the DC bus voltage, HESS only providing transient power to the loads).

\section{Methodology}

A more generic definition of efficiency, can be obtained through the integration of the instantaneous power along a given time (i.e. energy, $E$ ) of expression (1). This yields to the following expression where sub-index Loss stands for losses:

$$
\begin{gathered}
\eta_{d}=\frac{E_{O}}{E_{I}}=\frac{E_{I}-E_{\text {Loss }}}{E_{I}} \\
\eta_{d}=\frac{\int_{t=0}^{t=T} p_{I}(t) \cdot d t-\int_{t=0}^{t=T} p_{\text {Loss }}(t) \cdot d t}{\int_{t=0}^{t=T} p_{I}(t) \cdot d t}
\end{gathered}
$$

For the system operating in steady state, in rated conditions, (1) and (5) and show the same results. However, for a system evolving in transients, the energies involved in a given interval must be considered, as the efficiency varies depending on the operating conditions. It is assumed that the target system behaviour is defined between instants $\mathrm{t}=0$ and $\mathrm{t}=\mathrm{T}$. The idea is to calculate the energy losses along the evolution of the system in the typical operating conditions, including transient operation. Given that this evolution is a function of the control scheme implemented, the main goal of the work is to obtain an expression of the instant losses as a function of the control variables:

$$
p_{\text {Loss }}(t)=f\left(x_{1}(t), x_{2}(t), \ldots, x_{n}(t), p_{1}, p_{1} \ldots p_{m}\right)
$$

where $p_{\text {Loss }}(t)$ is the instant value of the power losses, $x_{1}, \ldots x_{n}$ are the $n$ control variables of the system, and $p_{1} \ldots p_{m}$ are the $m$ independent design parameters that are considered.

Once this expression is obtained, and once the evolution of the control variables is selected, the evaluation of the total energy loss that takes in the system upon these operating conditions can be calculated using (5). A key aspect to consider, is that the exact evolution of these control variables might not be known at the design stage. To solve this system, complex historic load profiles of the evolution of the system might be obtained. These profiles must have enough resolution and sampling rate as to effectively account for the complete system behaviour.

Even though this would provide accurate enough results, this kind of load profiles might not be available for all the applications. In addition, it might yield to a significant amount of calculations that can complicate the procedure. The approach followed in this work will be to select simple, yet representative transient profiles of the defined operational modes, that simplifies the comparison procedure. This allows for a comparative study that can enlighten the decision of the most suitable power topology for a given application. But also, it helps to select the best set of design parameters once a topology is chosen.

The proposed methodology will be tested in a HESS converter for two different possible topologies, each of them upon two given modes of operation.

\section{CASE OF STUdY: APPLICATION TO HYBRID ENERGY STORAGE SYSTEMS}

The forthcoming discussion considers the performance, in terms of efficiency, of a HESS as the one depicted in Fig. 1. The system under consideration has two energy storage devices, an electrochemical battery (EB) module intended for sustained power support, and a module of supercapacitors (SM) intended for fast-dynamics peak power support. At Fig. 1, the PECG is the Power Electronics Converter for Grid interface; the PEGL is the Power Electronics converter for Generation/Load and ESS accounts for Energy Storage System. Table I shows the main parameters of the HSS system.

Two different power topologies will be considered. The topology defined as Direct Parallel Connection (DPC) can be seen in Fig. 2.a. The alternative topology is the Series-Parallel Connection (SPC), depicted in Fig. 2.b. Both topologies have been deeply analysed in previous works [16] [17].

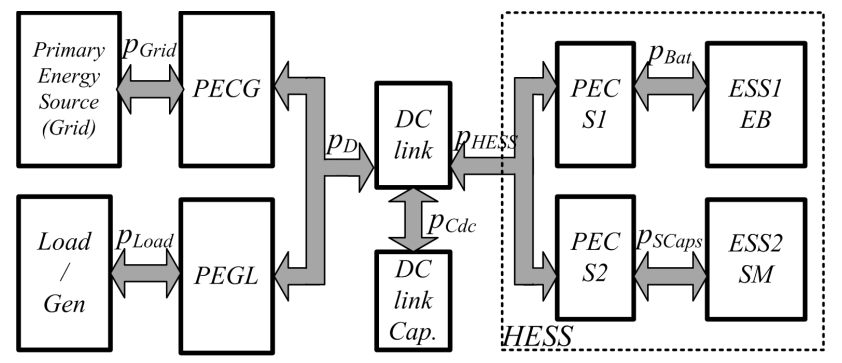

Fig. 1. Modeling approach in terms of the power flow balance in the considered Hybrid Energy Storage System (HESS).

TABLE I: RATED (NOMINAL) PARAMETERS OF THE SYSTEM UNDER STUDY

\begin{tabular}{ccc}
\hline \hline Symbol & Parameter & Value \\
\hline$V_{D C}$ & DC link Voltage & $600 \mathrm{~V}$ \\
\hline$V_{B A T}$ & Battery Voltage & $300 \mathrm{~V}$ \\
\hline$i_{\text {Bat }}$ & Battery Current (nom) & $20 \mathrm{~A}$ \\
\hline$V_{S C a p s}$ & SM Voltage (nom) & $60 \mathrm{~V}$ \\
\hline$i_{L S C a p s}$ & SM Current (nom) & $40 \mathrm{~A}$ \\
\hline$f_{S W}$ & Switching Frequency & $20 \mathrm{kHz}$ \\
\hline$P_{\text {NOM }}$ & Power of the converter & $8 \mathrm{~kW}$ \\
\hline
\end{tabular}

The theoretical expression of the losses needs to be calculated as a function of the control variables. For the considered application, the control scheme implemented is depicted in Fig. 2.c. The system power flows are controlled by current control loops at the storage systems, through standard PI regulators. The control variables for the battery and for the supercapacitor module will be the currents through the inductances $L_{B a t}$ and $L_{S C a p s}$, i.e. $i_{\text {LBat }}$ and $i_{\text {LSCaps }}$, respectively. In addition, there is a DC link control loop, however the small variations in the DC link due regular operation of the converter are considered not to affect significantly the efficiency performance of the system.

Each of those current control loops has a number of design parameters that tune the controllers. This work will assume a first order behaviour of the current loops, and therefore the main design parameters for each control loop will be the bandwidth of the controller. For any instant, (6) can be particularized for the target system as:

$$
p_{\text {Loss }}(t)=f\left(i_{\text {Lbat }}(t), i_{\text {LSCaps }}(t), V_{D C}, V_{\text {Bat }}, V_{\text {SCaps }}\right)
$$


where $V_{D C}, V_{B a t}$ and $V_{S C a p s}$ are the voltages at the DC link, at the battery and at the supercapacitor module.
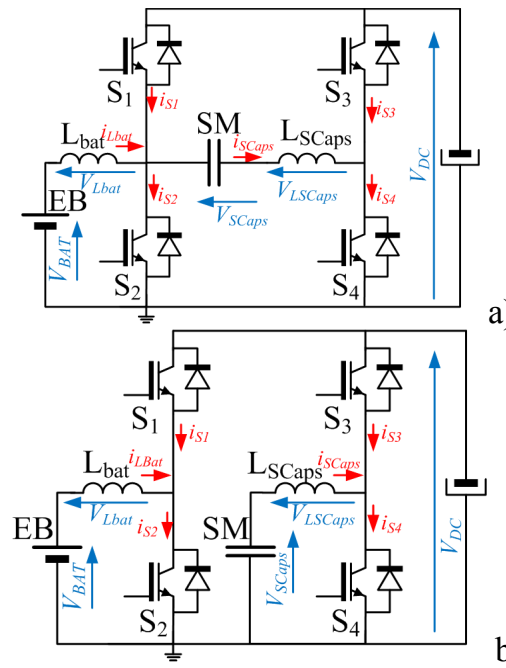

b)

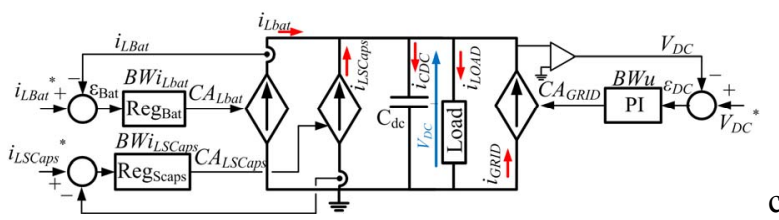

c)

Fig. 2.a) Direct Parallel Connection of two energy storage devices. b)

Series-Parallel Connection. c) Control scheme of the HESS considered

Initially, only these steady state losses will be calculated. In a second stage, the losses as a function of the system time evolution will be considered (i.e. as a function of the bandwidths involved).

\section{LOSSES IN STEADY STATE}

The total losses in the system considered are the switching and conduction losses at each of the switches, the magnetic losses at the inductors, as well as the DC link losses. The rest of the losses in the system will be disregarded. The expression of the switching losses at each switch has been modelled by:

$$
p_{S W}(t)=\left(V_{D C} \cdot I_{L o n} \cdot t_{T o n} \cdot E_{o n}+V_{D C} \cdot I_{L o f f} \cdot t_{T o f f} \cdot E_{o f f}\right) \cdot f_{S W}
$$

where $I_{\text {Lon }}$ and $I_{\text {Loff }}$ are the values of the instant currents through the inductor at the switching on and off instants (therefore considering the effect of the HF ripple), $f_{S W}$ is the switching frequency, $t_{\text {Ton }}$ and $t_{\text {Toff }}$ are the turn-on and turn-off switching intervals of the switch and $E_{\text {on }}$ and $E_{\text {off }}$ are switching energy values taken from the manufacturer's datasheet.

The conduction losses at each of the switches are given by:

$$
p_{C}(t)=V_{C E o n} \cdot I_{C a v g} \cdot D+R_{C E o n} \cdot I_{C r m s}^{2}
$$

where $V_{C E o n}$ and $R_{C E o n}$ are the collector to emitter on voltage and resistor of the considered IGBT given from the manufacturer's datasheet, and $I_{C a v g}$ and $I_{C r m s}$ are the average and $r m s$ values of the collector current.

The losses at the inductors have been calculated as:

$$
p_{L I}(t)=R_{L s e r} \cdot I_{\text {Lrms }}^{2}
$$

where $R_{\text {Lser }}$ is the series parasitic resistor of the inductor, and $I_{L r m s}$ is the $r m s$ value of the inductor current.

Finally, the losses at the DC link $\left(p_{L C d c}\right)$ can be considered by the ESR of the capacitors, although in VSI converter. These losses are usually low [4], and are given by:

$$
\text { "cp } \quad \text { "c } \quad \text { " } c I_{+}^{2} \text { c rms }
$$

where $E S R_{C D C}$ is the equivalent series resistance of the DC link capacitor, and $I_{C D C r m s}$ is the rms value of the current through the DC link capacitor.

Notice that each of the current and voltage values in the expressions (8)-(11) are a function of the instant value of the control parameters ( $\left.i_{L B a t}, i_{L S C a p s,} V_{D C}, V_{B a t}, V_{S C a p s}\right)$.

The final expression of the losses has been calculated as the addition of these partial components, for a given setup for the system defined in Table II. The resulting expressions are depicted in Fig. 3.a. These plots show an instant power losses map, as a function of currents $i_{\text {Lbat }}$ and $i_{\text {LScaps }}$ (horizontal and vertical axes, respectively). The darker the zone in the map, the lower the losses in steady state in that set of coordinates. Two different maps have been calculated, for each one of the two possible topologies considered, DPC (left) and SPC (right).

However, the idea underlying this work is to make a methodology for comparing different power topologies. In this sense, it is more interesting to depict a single instant power losses map, that accounts for the difference in the losses among the two possible options. This map is represented in Fig. 3.b. In this case, the lighter the zone in the map, the higher the losses difference, defined as:

$$
\Delta p_{\text {Loss }}=p_{\text {LossDPC }}-p_{\text {Loss } S P C}
$$

Therefore, the darker areas imply that there is a saving in the losses if SPC topology is used. In particular, the yellow lines mean that both losses are equal. This defines four regions in the losses map, two "light" areas (DPC is therefore preferred in terms of losses), and two "dark" regions (SPC provides less losses than DPC).

The next step is to define the typical operation of the system under consideration, as a trajectory in the losses map. If this trajectory falls completely in one of these areas, then the decision on the topology to use is obvious. On the case that these trajectories move through several of these areas, a more complex study must be carried out.

TABLE II: PARAMETERS OF THE CONVERTERS

\begin{tabular}{ccc}
\hline \hline Symbol & Parameter & Value \\
\hline$C_{D C}$ & DC link Capacitance & $2.2 \mathrm{mF}$ \\
\hline$E S R_{C D C}$ & ESR of DC link Capacitor & $<20 \mathrm{~m} \Omega$ \\
\hline$L_{\text {bat }}$ & Battery Converter Induct. & $2.1 \mathrm{mH}$ \\
\hline$R_{\text {Lbat }}$ & Series Resistance of $\mathrm{L}_{\text {bat }}$ & $0.2 \Omega$ \\
\hline$L_{\text {Scaps }}$ & Supercaps Converter Induct. & $350 \mu \mathrm{H}$ \\
\hline$R_{L S c a p s}$ & Series Resistance of $\mathrm{L}_{\text {Scaps }}$ & $0.2 \Omega$ \\
\hline$S_{X}$ & IGBT reference (IXYS) & MIXA60WB1200TEH \\
\hline \hline
\end{tabular}


However, the map of Fig. 3.b provides an initial guess of the relationship of operating values in which each of the two topologies under consideration presents a better efficiency performance (e.g. SPC will be preferred if the system operates for long intervals with supercapacitor currents from zero to around twice of the battery current, both with the same sign).

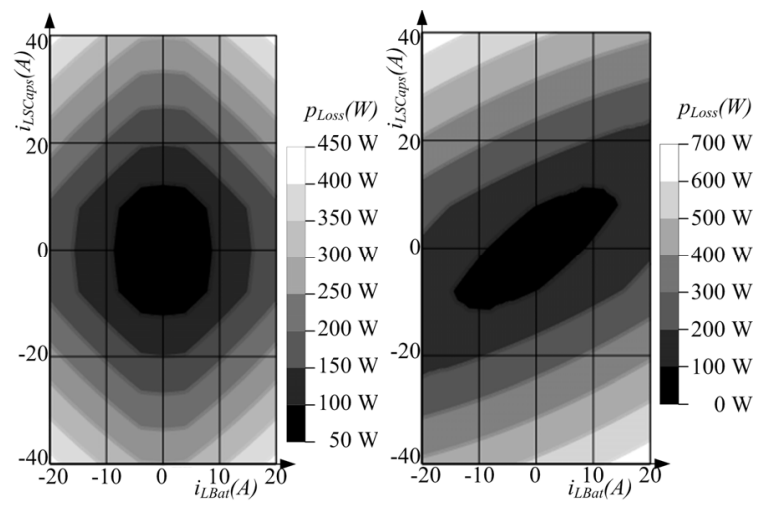

a)
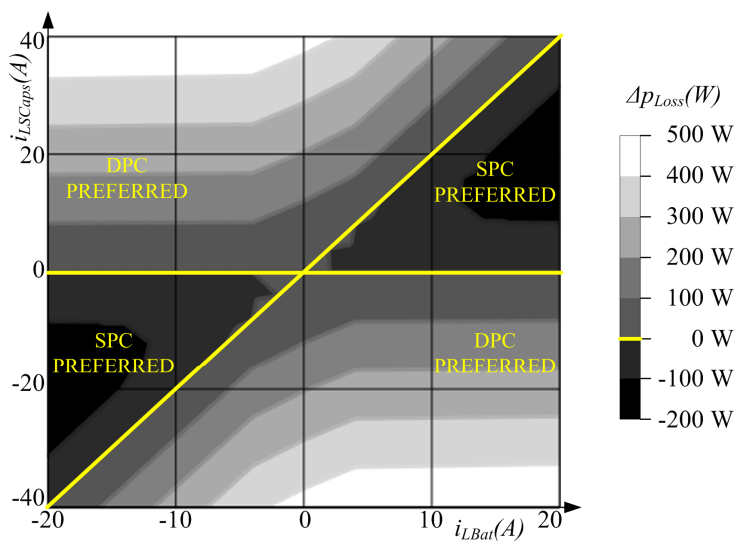

Fig. 3.a) Instant power losses map, as a function of battery and supercaps inductor currents, for DPC (left) and SPC (right) configuration. The darker the area, the lower the losses. b) Instant power losses subtraction map, as a function of the battery and supercaps inductor currents. The darker the area, the higher the losses savings in SPC (SPC losses smaller than DPC losses).

\section{DYNAMIC EVOLUTION: OpERATION MODES}

In general terms, the time dependence of the currents involved is a function, among other design parameters, of the bandwidth of the current control loops for the battery and supercaps, $B W i_{L b a t}$ and $B W i_{L S C a p s}$, respectively:

$$
\begin{gathered}
i_{\text {Lbat }}(t)=f\left(B W i_{\text {Lbat }}, t\right) \\
i_{\text {LSCaps }}(t)=f\left(B W i_{L S C a p s}, t\right)
\end{gathered}
$$

Therefore, the study of the efficiency of the system must consider these bandwidths as parameters. In addition, two different cases of operation will be defined. Finally, the performance in terms of losses of such cases of operation for the two considered topologies will be calculated. The considered cases are defined in Fig. 4, and are discussed ahead.

\section{A. Case A: Islanding operation}

This case accounts for a system in which initially the microgrid is working in islanding mode. Thus the HESS must support the full power to the loads as depicted in Fig. 4.a.
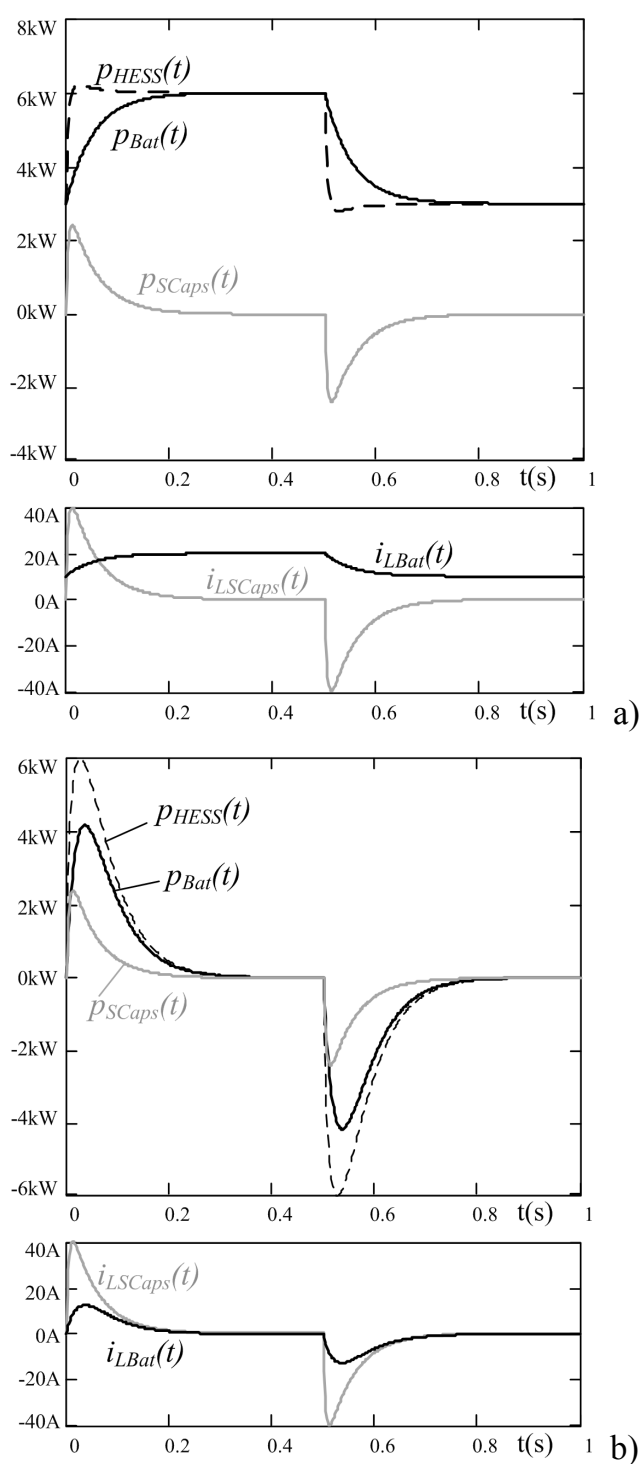

Fig. 4. Characterization of time evolution of battery and supercapacitor power and inductor current waveforms, for the three cases considered. a)

Case A: Islanding Operation. b) Case B: Grid-tied (transient support).

The typical operating profile selected consists of repetitive 3 $k W$ alternative power steps, as can be seen in Fig. 4.a. At each of these steps, the battery provides a first order response, with a given bandwidth, up to the corresponding current command step. In this case, the steps take place each $0.5 \mathrm{~s}$, and this base bandwidth has been selected of $3 \mathrm{~Hz}$ for illustration. On the other side, the supercapacitor provides the required power peaks as to obtain the desired power through the loads. Therefore, the corresponding current reference starts with a high peak value (around $40 \mathrm{~A}$ ), and decays to zero after some time, also characterized by a given bandwidth (the same base bandwidth of $3 \mathrm{~Hz}$ ). Therefore, the SM subsystem helps to provide initial pulsed power, whereas the battery provides a continuous power in steady state.

The trajectory on the losses map of the Cartesian coordinates given by the evolution of $i_{\text {LSCaps }}$ and $i_{\text {Lbat }}$, is presented in Fig. 5.a. Different relative bandwidths in the current controllers are shown, in order to illustrate the dependency of these trajectories on the control design parameters. The red curve depicts the exact case seen in Fig. 4.a. The blue trajectory shows a case similar to the red one, keeping the base bandwidth for the battery current 
controller, but with a bandwidth in the supercaps inductor current 5 times slower than in the previous case. Finally, the green line shows also a similar case to the red one, in this case with the supercaps current bandwidth 5 times faster. The time step, $t_{\text {step }}$, between two consecutive dots in the trajectories is constant $(0.2 \mathrm{~ms})$. Therefore, it can be seen how the values of the control design parameters, in this case both current bandwidths, significantly affect the evolution of the losses in the system. In order to decide upon the optimal topology for this case A, the detailed numerical calculations for the total energy loss must be carried out. These calculations will be discussed in the next section.

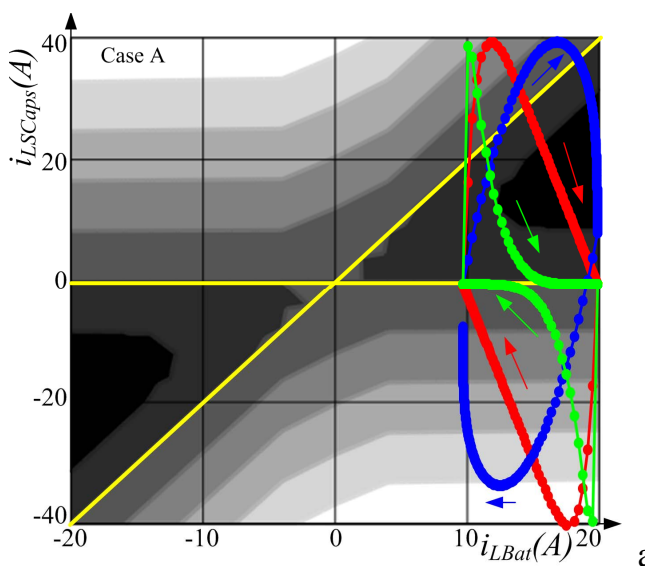

a)

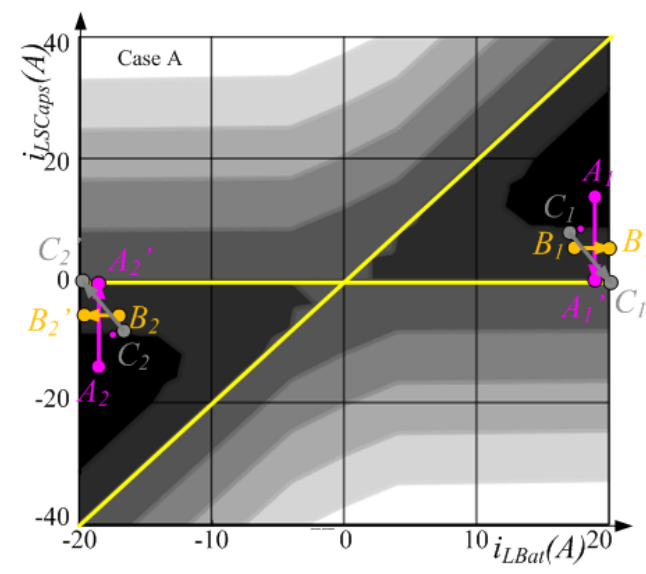

b)

Fig. 5. a) Trajectories over the instant power losses subtraction map for different relative bandwidths in the current controllers, for case A. (red: cases depicted in Fig. 4; blue: same than red, with BW for supercaps 5 times slower; green: same than red, with BW for supercaps 5 times faster.)

b) Evolution of the centroids of the trajectories, as a function of bandwidth values in the current controllers for case A (magenta: keeping $B W i_{L b a t}$ constant, increasing $B W i_{L S C a p s}$, orange: keeping $B W i_{L S C a p s}$ constant, increasing $B W i_{\text {Lbai }}$; green: Increasing both bandwidths, keeping $B W i_{L S C a p s} 10$ times faster)

Another way to visualize this trend is shown in Fig. 5.b. As mentioned, each trajectory, defined by the bandwidths $B W i_{L b a t}$, and $B W i_{L S C a p s}$, is represented by a set of points in the XY plane, being $\mathrm{X}$ the battery current and $\mathrm{Y}$ the supercaps current. These points can thus be represented by a vector:

$$
\vec{r}_{l}=\left(x_{i}, y_{i}\right)=\left(i_{\text {LBat } i}, i_{\text {LSCaps } i}\right)
$$

being $\vec{r}_{\mathrm{i}}$ the position vector of each point in the sequence.

Considering that each point in the trajectory is given at a constant sampling time, then the centroid of the set of points can be calculated following:

$$
\overrightarrow{R_{C T R D}}=\frac{\sum_{i} \overrightarrow{r_{l} \cdot t_{s t e p}}}{T}
$$

being $\overrightarrow{R_{C T R D}}$ the vector of the centroid, and $\mathrm{T}$ the total time of the signal.

This centroid has been represented in Fig. 5.b, for three different conditions. The evolution from point $\mathrm{A}_{1}$ to $\mathrm{A}_{1}$ ' (and $\mathrm{A}_{2}$ to $\mathrm{A}_{2}$ ', for the negative cycle), shows how the centroid is displaced when $B W i_{L b a t}$ is kept constant and equal to the $3 \mathrm{~Hz}$ reference value, while $B W i_{L S C a p s}$ is increased from $1 / 10$ to 10 times this value (magenta trace). As it can be seen, initially the SPC configuration is better from the point of view of the losses, but as $B W i_{L S C a p s}$ increases, evolves to a neutral situation.

The orange trace shows the trend when $B W i_{L S C a p s}$ is kept constant, and $B W i_{L b a t}$ increases ( $\mathrm{B}_{1}$ to $\mathrm{B}_{1}$ ' and $\mathrm{B}_{2}$ to $\left.\mathrm{B}_{2}{ }^{\prime}\right)$. For this particular case, the evolution does not mean any improvement from the point of view of the losses.

Obviously in all this discussion, the feasible limit values for the bandwidths must be considered, as not all the points in the trajectory would have a practical meaning. In this sense, the grey trace shows a more realistic situation, in which the bandwidth of the battery converter is always 10 times slower than the bandwidth of the supercaps, and both values increase from $\mathrm{C} 1$ to $\mathrm{C} 1$ ' (and from C2 to C2'). It can be seen how in all cases SPC will provide less losses, however the slower the bandwidth, the better.

\section{B. Case B: Grid-tied, transient support}

Figure 6 shows the grid tied mode configuration. This case accounts for a system in which the microgrid operates in gridtied mode, and the HESS provides only transient power at each load steps. Eventually, the power delivered by the HESS reaches zero. The current evolutions considered for the battery and the supercaps are depicted in Fig. 4.b. In this case, the power delivered by the supercaps is the same as in case A. On the other hand, the battery current has been implemented with a combination of a LPF and a HPF, also at $3 \mathrm{~Hz}$, that forces the steady state of the battery current to be zero. For these current shapes, a similar procedure has been followed for this case. The trajectories for three different $B W i_{L S C a p s}$ are shown in Fig. 6.a. Again, the system performance clearly depends on the dynamic design parameters.

The centroids have been also calculated for this situation, and are depicted in Fig. 6.b. The magenta line shows the displacement of the centroid for the same conditions than in case A (constant battery converter bandwidth, increasing supercaps bandwidth). It can be seen that, in this case, increasing BWiLScaps yields to a significant decrease in the losses, actually passing from a situation in which DPS is preferred (points $A_{1}$ and $A_{2}$ ) to a situation in which SPC is the best option (points $A_{1}$, and $A_{2}^{\prime}$ ).

On the other hand, the orange trace shows exactly the opposite trend, for the case in which the bandwidth of the supercaps converter is kept constant, while increasing the battery converter bandwidth. The initial situation considers SPC as the best option (points $\mathrm{B}_{1}$ and $\mathrm{B}_{2}$ ), however increasing the battery bandwidth yields to an increase in the SPC losses up to the point that DPC is finally preferred (points $\mathrm{B}_{1}$ ' and $\mathrm{B}_{2}{ }^{\prime}$ ).

Finally, the grey trace shows the case that $B W i_{L S C a p s}$ is one order of magnitude higher than $B W i_{\text {Lbat }}$. In this case, SPC is 
always preferred, but again the faster the system is, the higher the losses at the SPC configuration compared to DPC.
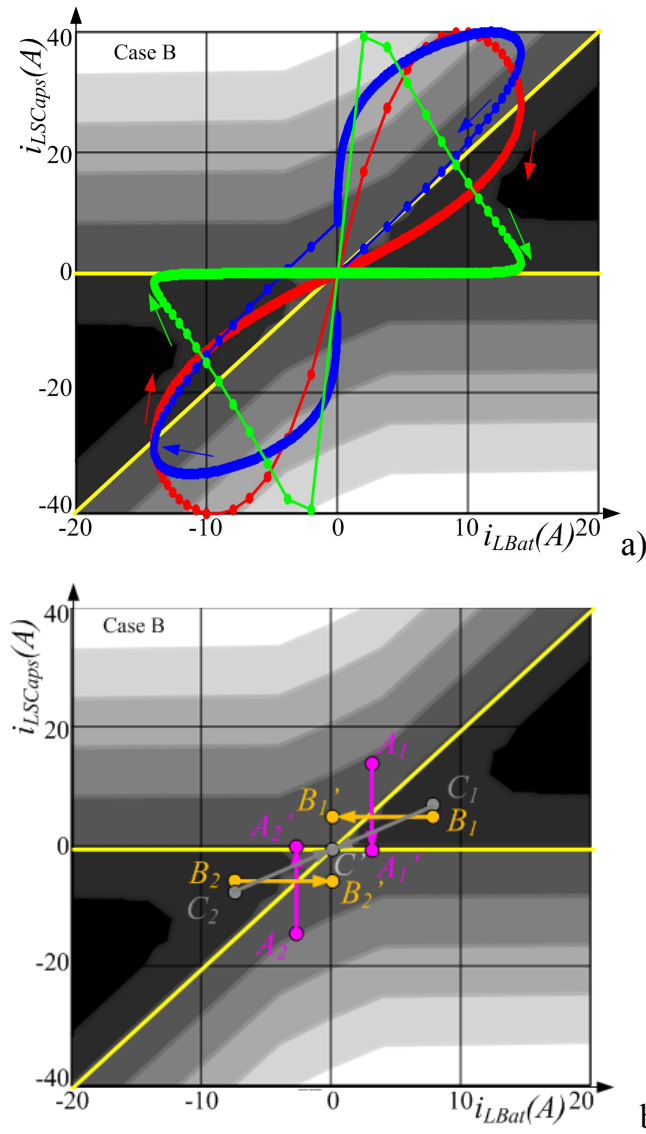

b)

Fig. 6. a) Trajectories over the instant power losses subtraction map for different relative bandwidths in the current controllers, for case B. (red: cases depicted in Fig. 4; blue: same than red, with BW for supercaps 5 times slower; green: same than red, with BW for supercaps 5 times faster.)

b) Evolution of the centroids of the trajectories, as a function of bandwidth values in the current controllers for case B (magenta: keeping $B W i_{L b a t}$ constant, increasing $B W i_{L S C a p s}$; orange : keeping $B W i_{L S C a p s}$ constant, increasing $B W i_{L b a t} ;$ green: Incerasing both bandwidths, keeping $B W i_{L S C a p s} 10$ times faster)

This analysis of the centroid evolution can be used in order to have a first approach of the expected behaviour of the converters and the efficiency, as to initially settle the operating conditions of the system. However, in order to refine the results, a complete computing of the results must be carried out.

\section{COMPUTING THE EFFICIENCY}

The complete calculation of the losses, for each of the configurations (SPC or DPC) has been carried out for both cases of operation (cases A and B), using as parameters the bandwidths of the battery and supercaps current loops.

The value of the energy loss has been calculated, and have been depicted in Fig. 7. However, the information of the energy losses evolution does not give idea of the relative importance of these variations to the overall energy processed by the system. Thus, this calculation must be compared with the overall energy converted by the system.

In order to address this issue, the dynamic efficiency, $\eta_{d}$, as defined in (5), has been computed, for the full waveform periods as defined in Fig. 4. This parameter has been represented in the charts of Fig. 8.
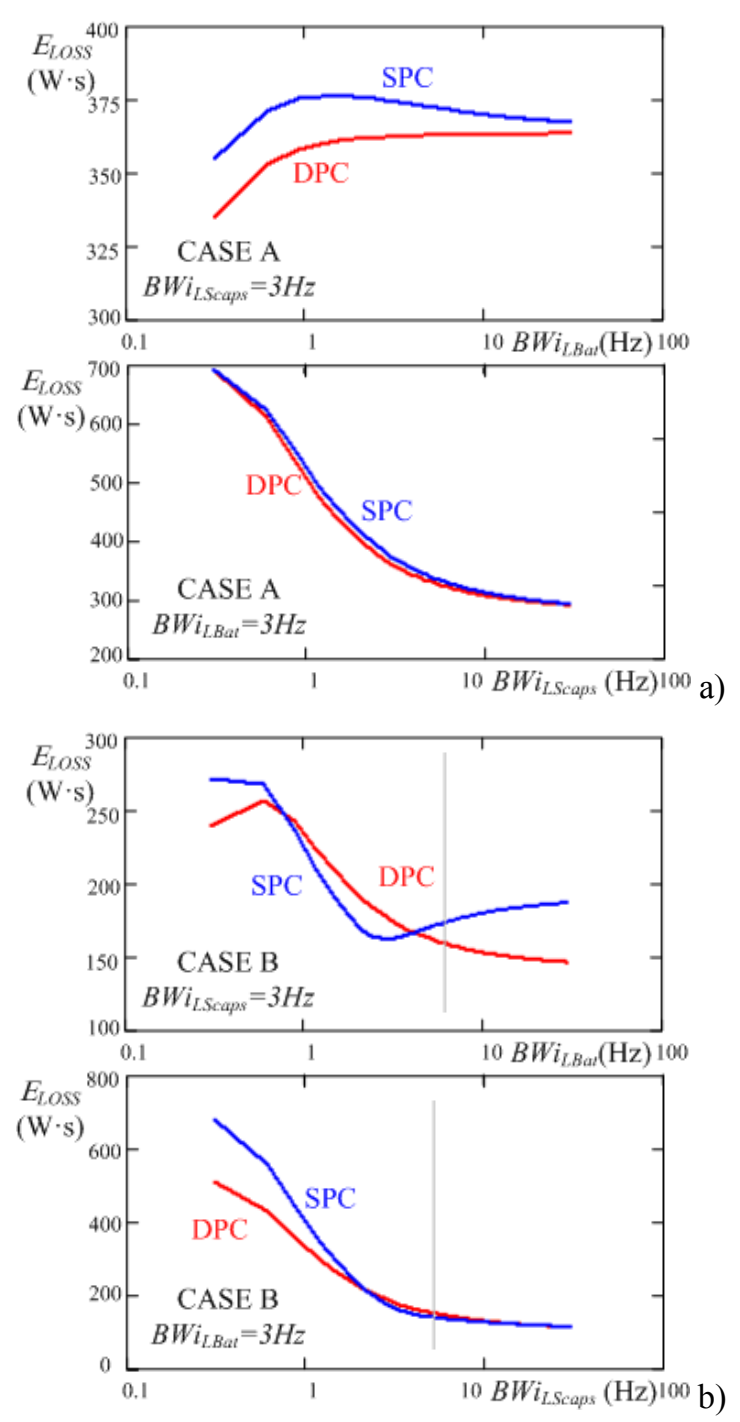

Fig. 7. Evolution of the Energy losses, $\mathrm{E}_{\mathrm{LOSS}}$ (in W.s) calculated with the trajectories defined for: a) case A, and b) case B. Upper plots are calculated for a fixed $B W i_{L S C a p s}=3 \mathrm{~Hz}$, for different $B W i_{L b a t}$ values. Lower plots are calculated for a fixed $B W i_{L b a t}=3 \mathrm{~Hz}$, for different $B W i_{L S c a p s}$ values

The overall dynamic efficiency of the system can be seen, as a function of the control parameters, i.e. keeping the battery bandwidth in the base value of $3 \mathrm{~Hz}$ and varying the supercapacitor bandwidth (from 0.3 to $30 \mathrm{~Hz}$ ). This has been carried out, at each of the cases considered (A and B), for both topological configurations (DPC and SPC). It can be noticed how there are some sets of conditions in which the efficiency at one topology is higher than the other one, therefore enabling for a selection of the optimal operating point for given set of operational constraints of the HSS. For instance, for the particular case A, depicted in Fig. 4.a, in terms of the efficiency performance, there is practically no difference using either configuration (DPC or SPC). However, for case B, there is a margin of design at which SPC presents more efficiency than DPC; away from this margin DPC presents better efficiency.

This behaviour is consistent and can be deduced from the efficiency plots at Fig. 5 (right), where the red line (both supercaps and battery current bandwidths equal to $3 \mathrm{~Hz}$ ) falls mainly on the "SPC preferred" region; however both the green and blue plots (supercaps current bandwidth $15 \mathrm{~Hz}$ and $0.6 \mathrm{~Hz}$, respectively) flow through the "DPC" preferred area. 

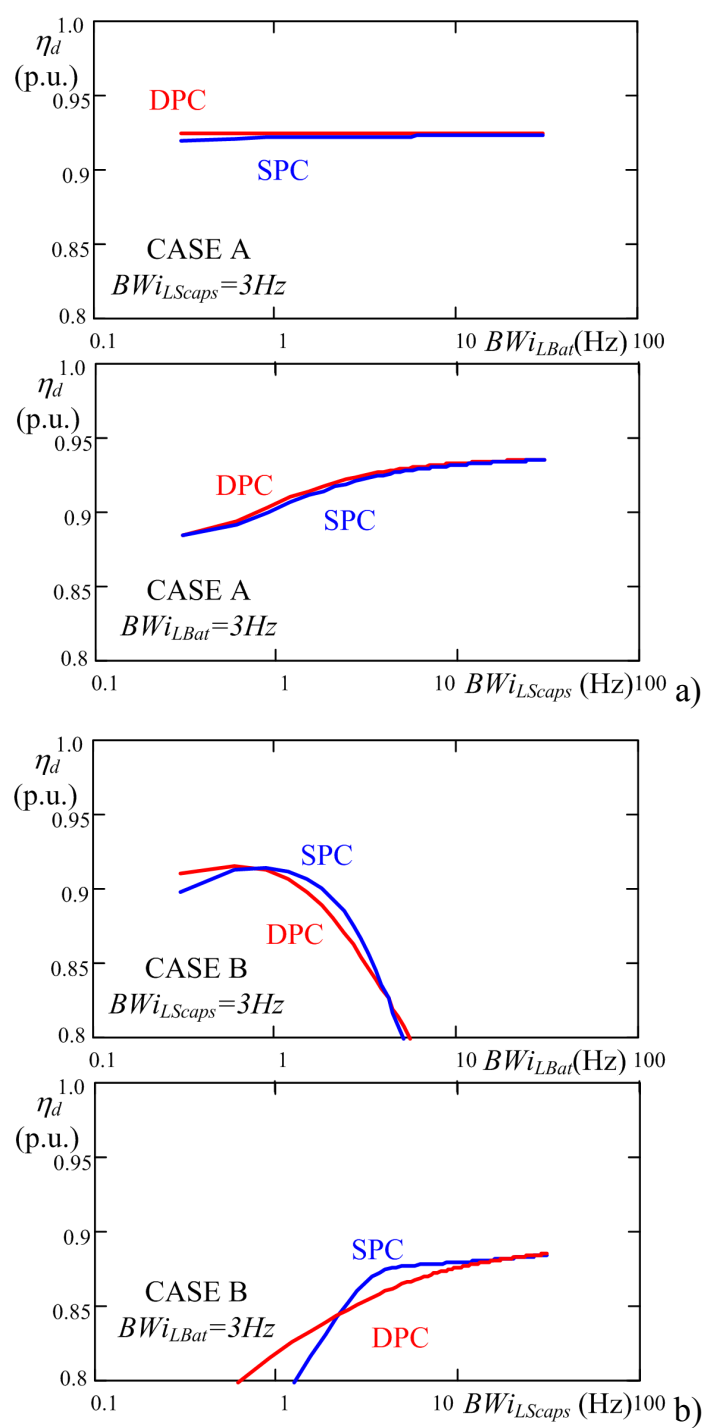

Fig. 8. Evolution of the dynamic efficiency, $\eta_{\mathrm{d}}$ (in p.u.) calculated with the trajectories defined for: a) case A, and b) case B. Upper plots are calculated for a fixed $B W i_{L S C a p s}=3 \mathrm{~Hz}$, for different $B W i_{L b a t}$ values. Lower plots are calculated for a fixed $B W i_{L b a t}=3 \mathrm{~Hz}$, for different $B W i_{L S c a p s}$ values

\section{CONCLUSIONS AND FUTURE DEVELOPMENTS}

A methodology for optimal dynamic efficiency performance design of power converters has been presented. This methodology has been illustrated by applying it to the study of the performance of power electronic converters for hybrid energy storage systems.

Once the system is defined (nominal values, case of operation, etc.), the shown loss charts, centroid evolutions and dynamic efficiency plots can be calculated, and used as an aid tool for decision making, in order to select among different options of topologies.

Even more, once a topology is finally chosen, the system can be optimized, in terms of losses, by adjusting the bandwidths of the control loops (obviously, within the range of dynamic requirements and constraints of the system).

Future developments include the testing of this methodology with real load profiles in the system, as well as an experimental validation of the methodology.

\section{REFERENCES}

[1] H. H. Sathler, L. H. Sathler, F. L. F. Marcelino, T. R. de Oliveira, S. I. Seleme and P. F. D. Garcia, "A comparative efficiency study on bidirectional grid interface converters applied to low power DC nanogrids," 2017 Brazilian Power Electronics Conference (COBEP), JUIZ DE FORA, Brazil, 2017, pp. 1-6. doi: 10.1109/COBEP.2017.8257219

[2] G. Chen and X. Cai, "Adaptive Control Strategy for Improving the Efficiency and Reliability of Parallel Wind Power Converters by Optimizing Power Allocation," in IEEE Access, vol. PP, no. 99, pp. 1-1. doi: 10.1109/ACCESS.2018.2789421

[3] K. N. D. Malamaki, C. S. Demoulias and K. O. Oureilidis, "Analytical calculation of the PV converter efficiency curve at non-unity power factors," 2017 52nd International Universities Power Engineering Conference (UPEC), HERAKLION, Crete, Greece, 2017, pp. 1-6. doi: 10.1109/UPEC.2017.8231924

[4] E. P. Wiechmann, P. Aqueveque, R. Burgos and J. Rodriguez, "On the Efficiency of Voltage Source and Current Source Inverters for High-Power Drives," in IEEE Transactions on Industrial Electronics, vol. 55, no. 4, pp. 1771-1782, April 2008. doi: 10.1109/TIE.2008.918625

[5] J. A. Galaviz-Aguilar, H. C. Chang, F. Martinez-Rodriguez, P. Roblin and J. C. Nunez-Perez, "Measurement of dynamic power dissipation and estimation of effective dynamic efficiencies in an LTE chireix PA," 2016 88th ARFTG Microwave Measurement Conference (ARFTG), Austin, TX, 2016, pp. 1-4. doi: 10.1109/ARFTG.2016.7839717

[6] G. He, Q. Chen, C. Kang and Q. Xia, "Optimal operating strategy and revenue estimates for the arbitrage of a vanadium redox flow battery considering dynamic efficiencies and capacity loss," in IET Generation, Transmission \& Distribution, vol. 10, no. 5, pp. 1278-1285, 47 2016. doi: 10.1049/iet-gtd.2015.0373

[7] K. Ishaque and Z. Salam, "Dynamic Efficiency of Direct Control Based Maximum Power Point Trackers," 2014 5th International Conference on Intelligent Systems, Modelling and Simulation, Langkawi, 2014, pp. 429434. doi: 10.1109/ISMS.2014.79

[8] Z. Giovanni, C. Emiliano, F. Andrea and R. Carlo, "New control techniques for UPS dynamic efficiency optimisation," INTELEC 2009 - 31st International Telecommunications Energy Conference, Incheon, 2009, pp. 1-5. doi: 10.1109/INTLEC.2009.5351967

[9] J. Rodríguez, J. Dixon, J. Espinoza, J. Pontt, and P. Lezana, "PWM regenerative rectifiers: State of the art," IEEE Trans. Ind. Electron., vol. 52, no. 1, pp. 5-22, Feb. 2005.

[10] J.Rodríguez,J.Pontt,N.Becker,andA.Weinstein, "Regenerativedrivers in the megawatt range for high-performance downhill conveyors," IEEE Trans. Ind. Appl., vol. 38, no. 1, pp. 203-210, Jan./Feb. 2002.

[11] California Energy Commission (CEC) Emerging Renewables Program (ERP) Guidebook, 4th Edition, posted on the web in January 2005, http://www.energy.ca.gov/2005publications/CEC-300-2005001/CEC-300-2005-001-ED4F.PDF

[12] Standard IEC 61683:1999, "Photovoltaic systems-Power conditionersProcedure for measuring efficiency," International Electrotechnical Commission, 1999.

[13] B. Brooks and C. M. Whitaker, "Guideline for the use of the Performance Test Protocol for Evaluating Inverters Used in Grid-Connected Photovoltaic Systems," California Energy Commission, Feb. 2005.

[14] J. Newmiller, W. Erdman, J. S. Stein and S. Gonzalez, "Sandia Inverter Performance Test Protocol efficiency weighting alternatives," 2014 IEEE 40th Photovoltaic Specialist Conference (PVSC), Denver, CO, 2014, pp. 0897-0900. doi: 10.1109/PVSC.2014.6925058

[15] Y. H. Kim, J. W. Jang, S. C. Shin and C. Y. Won, "Weighted-Efficiency Enhancement Control for a Photovoltaic AC Module Interleaved Flyback Inverter Using a Synchronous Rectifier," in IEEE Transactions on Power Electronics, vol. 29, no. 12, pp. 6481-6493, Dec. 2014. doi: 10.1109/TPEL.2014.2306261

[16] R. Georgious, J. Garcia, A. Navarro, S. Saeed and P. Garcia, "SeriesParallel Connection of Low-Voltage sources for integration of galvanically isolated Energy Storage Systems," 2016 IEEE Applied Power Electronics Conference and Exposition (APEC), Long Beach, CA, 2016, pp. 35083513 . doi: 10.1109/APEC.2016.7468372

[17] J. Garcia, R. Georgious, P. Garcia and A. Navarro-Rodriguez "Non-Isolated High-Gain Three-Port Converter for Hybrid Storage Systems", ECCE 2016. 